\title{
A systematic review on bowel management and the success rate of the various treatment modalities in spina bifida patients
}

\author{
SV Velde, SV Biervliet, RD Bruyne and MV Winckel
}

Study design: Systematic review.

Objectives: To determine the different treatment modalities aimed at achieving fecal continence in spina bifida (SB) patients and their effectiveness.

Setting: International literature.

Method: Electronic databases were searched ('Pubmed', 'Web of science', 'CINAHL' and 'Cochrane') identifying studies published since the mid-eighties and screened for relevance according to the Centre for Reviews and Dissemination procedure guidelines. A total of 37 studies were selected for inclusion.

Results: Studies on toilet sitting, biofeedback, anal plug, retrograde colon enemas (RCE) and antegrade colon enemas were found. Fecal continence was achieved in $67 \%$ of SB patients using conservative methods $(n=509)$. In patients using RCE ( $n=190)$ an $80 \%$ continence rate was reached. Patients following surgical treatment $(n=469)$ reached an $81 \%$ continence rate, however, $23 \%$ needed redo surgery because of complications. Better fecal continence was associated with an improved quality of life, which was negatively influenced by the amount of time spent on bowel management.

Conclusion: Evidence favors an individually tailored stepwise approach with surgery as a final step in case of failure of conservative measures. Continued specialized support throughout life remains important to maintain continence. Cross-over and comparative trials are needed in order to optimize treatment.

Spinal Cord (2013) 51, 873-881; doi:10.1038/sc.2013.123; published online 15 October 2013

Keywords: spina bifida; fecal pseudo-continence; bowel management; constipation

\section{INTRODUCTION}

Spina bifida (SB) or meningomyelocele is a complex neuroembryological disorder resulting from a variable degree of incomplete closure of the posterior neural tube. Clinical presentation is highly variable and depends on the localization of the defect along the spinal cord and the degree of incomplete closure. These patients present with a spectrum of impairments but the primary functional deficits are lower-limb paralysis and sensory loss, bladder and bowel dysfunction and cognitive dysfunction. ${ }^{1}$

In the majority of patients the lower regions of the spine are affected, resulting in dysfunction of the distal gastrointestinal tract: rectum, anus and anal sphincter. Voluntary control of defecation requires normal rectal sensation, peristalsis and normal anal sphincter function. Two primary involuntary reflexes, the intrinsic and parasympathic reflex, located at sacral level 2-4, initiate defecation. The pudendal nerve, responsible for voluntary defecation, controls the opening and closing of the external anal sphincter. Nerve damage above the S2 level will impair both involuntary reflexes and pudendal nerve function. Loss of the involuntary reflexes perturbs rectal sensation and initiation of defecation. Damage of the pudendal nerve leads to partial or total loss of the voluntary sphincter control. Perturbation of these processes will result in bowel incontinence, which is often associated with constipation in SB patients. ${ }^{2,3}$ Fecal incontinence in SB patients is reported to be present in $28-53 \%$, regardless of the therapy used. ${ }^{4-7}$
Fecal and urinary incontinence importantly affect quality of life (QoL) in SB patients and form a major barrier to attending school, obtaining employment and sustaining relationships. Krogh et al. ${ }^{4}$ report that $66 \%$ of SB patients older than 6 years with fecal incontinence perceive incontinence as having a negative influence on their social activities. Lie et al. ${ }^{8}$ report that $75 \%$ of SB patients with urinary incontinence regard incontinence as a stress factor.

\section{REVIEW QUESTION}

A systematic literature review was performed regarding treatment modalities for constipation and/or fecal incontinence in SB patients and their success rate. Treatment success is defined as fecal continence that corresponds to stool losses less than once a month.

\section{REVIEW METHOD}

The review is performed following the guidelines of the Centre for Reviews and Dissemination procedures. ${ }^{9}$

Study selection

Inclusion criteria:

- Original papers with full paper available

- Written in English

- SB patient cohort larger than 20

- SB patients using any form of bowel management 


\section{Exclusion criteria:}

- No data on outcome of treatment for SB patients

- Review, opinion or editorial pieces

\section{Study identification}

The following databases were used for study identification: 'Pubmed' and 'Web of science', 'CINAHL' and 'Cochrane' database. A search was performed in March 2013. The following Mesh terms were used: 'spina bifida and bowel management', 'spina bifida and fecal incontinence', 'spina bifida and enema' 'myelomeningocele and bowel management', 'myelomeningocele and fecal incontinence' and 'myelomeningocele and enema'. The search covered studies published since 1986 as this was the start of important bowel management evolutions, with the introduction of retrograde colon enemas (RCE) by Shandling $^{10}$ and some years later the antegrade colon enema (ACE) by Malone. ${ }^{11}$

This database search resulted in a selection of 37 papers (see flowchart). ${ }^{4-7,10,12-43}$

A limitation of the review is the lack of search in gray literature as well as the absence of expert contact to attain more information on the subject.

Data extraction was performed independently by two authors to avoid selection bias in the process. From the full copies retrieved, two articles were not retained by both authors.

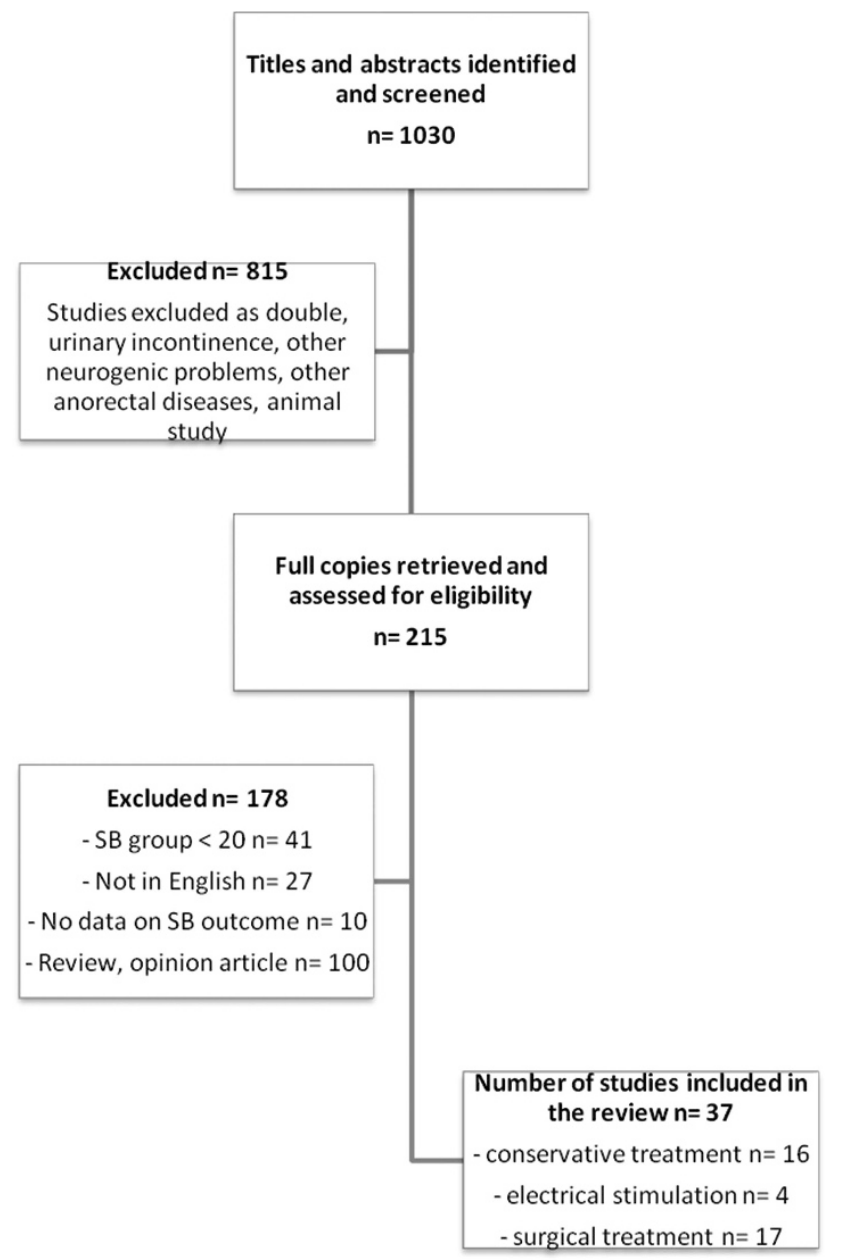

\section{RESULTS}

A total of 37 studies were included in the review. ${ }^{4-7,10,12-43}$

Most studies were observational studies with retrospective or prospective data on SB patient cohorts in a single or multiple center setting. Only two were randomized clinical trials on the use of electrical stimulation, of which one was double-blind controlled ${ }^{23}$ and one was patient-blinded. ${ }^{26}$ All studies had a low grade of recommendation. ${ }^{44}$

Clinical data were collected using question-

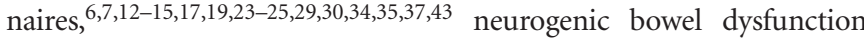
scales, ${ }^{20,26,45}$ QoL questionnaires, ${ }^{4,16,21,22,27,28,31,32,38,41}$ or child behavior checklists. ${ }^{4,31}$ The collection methods were interviews during follow-up visits $^{6,15,20,25,43}$ or telephone contacts ${ }^{4,7,12,17,23,32,38}$ or both. ${ }^{16,31,37,41}$

The follow-up period of SB patients after starting bowel management varied from $3,{ }^{15,19,20,22,23,41,42}$ over $12,12,13,21,25,31$

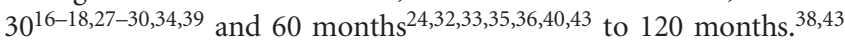

To explore different treatment modalities for bowel management in $\mathrm{SB}$ and their effectiveness the studies were grouped according to the treatment modality: conservative bowel management (16 studies), electrical stimulation (4 studies) and non-conservative (surgical) bowel management (17 studies).

\section{Conservative bowel management}

The studies on conservative bowel management are summarized in Table 1 . From the 16 studies, 1 reported on toilet sitting, 2 on biofeedback, 1 on anal plug use, 4 on a stratified treatment strategy and 8 on RCE.

Most studies on bowel management used the above-mentioned strict continence definition (no stool loss or stool loss less than once a month). ${ }^{4,6,12,16-21}$ Some studies gave no definition ${ }^{7,13,15}$ or used a lessstrict definition. ${ }^{5,10,14,22}$ The results for fecal pseudo-continence achievement using conservative bowel management varied from $36^{13}$ to $100 \% .^{10}$ In order to calculate the combined continence rate, only the studies using the strict continence definition of stool losses less than once a month were used. Fecal pseudo-continence was achieved in 67\% (341/509).

Looking only at the studies on RCE $(n=8)$, fecal pseudocontinence was achieved in $80 \%(144 / 190)$. Two studies reported on the effect of RCE on constipation. Mattsson et al. ${ }^{19}$ reported absence of constipation when using RCE, whereas Ausili et al. ${ }^{20}$ reported $60 \%(36 / 60)$ constipation relief using RCE. In both studies, all SB patients suffered from constipation at the start. The irrigation fluid used was saline in $3,{ }^{10,16,18}$ tap water in $4^{17,19,21,22}$ and one did not mention the fluid type used. ${ }^{20}$ The irrigation volumes used were sometimes fixed body weight-related quantities $\left(20 \mathrm{ml} \mathrm{kg}^{-1}\right)^{10,16,17}$ or varied between $300^{19}$ and $616 \mathrm{ml}^{21}$

The time spent on treatment was not often reported ${ }^{7,12-15,17,20}$ and if reported varied from $15^{5,10,16,21,22}$ over $30 \mathrm{~min}^{18}$ to $60 \mathrm{~min} .{ }^{19} \mathrm{Krogh}$ et $a .^{4}$ described a threefold increase in treatment time when comparing RCE with digital evacuation.

Although achieving fecal pseudo-continence is the primary treatment goal, patient satisfaction is an important secondary goal. Results on satisfaction could not be compared as all reporting studies used different questionnaires and scales. Some studies reported an improvement of satisfaction or a high satisfaction rate associated with successful bowel management. ${ }^{16,17,19-21}$ The study of Shoshan et al. ${ }^{15}$ described a significant reduction of daily life impairment by fecal incontinence after bowel management intervention. Finally, implementing a stepwise fecal incontinence treatment protocol improved fecal pseudo-continence and in parallel resulted in better socialization, less need for support by the caregiver and less negative 
Table 1 Summary of papers on conservative bowel management in SB patients

\begin{tabular}{|c|c|c|c|c|c|c|}
\hline Author & Sample size, age, type & FU/TT & Fl definition & Study design & Results-effectiveness & Results-satisfaction \\
\hline ng et al. ${ }^{12}$ & $\begin{array}{l}n=40 \mathrm{SB} \\
\text { Age: } 18 \text { months- } 29 \text { years } \\
\text { LL: } 12 \mathrm{~T}, 25 \mathrm{~L}, 2 \mathrm{~S} \\
\mathrm{FI}: 35 / 40 \\
\text { Constipation: } \mathrm{nm}\end{array}$ & $\begin{array}{l}\text { FU: } 15 \mathrm{~m} \\
\text { TT: not men- } \\
\text { tioned }(\mathrm{nm})\end{array}$ & $\begin{array}{l}\text { Stool loss < once } \\
\text { per month }\end{array}$ & $\begin{array}{l}\text { Single center } \\
\text { Retrospective chart review } \\
\text { Prospective bowel program: daily, } \\
\text { regular, consistently timed, reflex- } \\
\text { triggered bowel evacuation } \\
\text { Phone FU every } 2 \text { weeks and dur- } \\
\text { ing FU visits }\end{array}$ & $\begin{array}{l}\text { Continence: start 5/40 (12,5\%), } \\
15 \mathrm{~m}: 24 / 40(60 \%) \\
24 / 40 \text { compliant, } 19 / 24(79 \%) \\
\text { continent } \\
11 / 40 \text { non- compliant, none con- } \\
\text { tinent }(P<0.0001) \text {. }\end{array}$ & Not evaluated \\
\hline $\begin{array}{l}\text { Whitehead } \\
\text { et al. }{ }^{13}\end{array}$ & $\begin{array}{l}n=33 \mathrm{SB} \\
\text { Age: } 5-16 \text { years } \\
\text { LL: T10-S2 no mental } \\
\text { impairment } \\
\text { FI: } n m \\
\text { Constipation: } \mathrm{nm}\end{array}$ & $\begin{array}{l}\text { FU: } 12 \mathrm{~m} \\
\text { TT: } \mathrm{nm}\end{array}$ & Not defined & $\begin{array}{l}\text { Single center } \\
\text { Prospective-controlled trial. } \\
\text { Daily symptom log, } 1 \mathrm{~m} \text { before and } \\
\text { during } \\
19 \text { SB behavior modification: } 10 \\
\text { times toilet sitting every evening } \\
14 \text { biofeedback every } 2 \text { weeks and } \\
\text { behavior modification. }\end{array}$ & $\begin{array}{l}\text { Biofeedback: (before) } 5.38-1.93 \\
\text { ( } 12 \mathrm{~m} \text { ) accidents per week } \\
5 / 14 \text { (36\%) continent } \\
\text { Behavior: (before) } 5.77-2.3 \\
\text { ( } 12 \mathrm{~m} \text { ) accidents per week } \\
4 / 19 \text { (21\%) became continent. } \\
\text { No significant difference between } \\
\text { groups }\end{array}$ & Not evaluated \\
\hline $\begin{array}{l}\text { Ponticelli } \\
\text { et al. }{ }^{14}\end{array}$ & $\begin{array}{l}n=73 \text { SB ( } 67 \text { congeni- } \\
\text { tal) } \\
\text { Age: } 7-25 \text { years } \\
\text { LL: L5-S1 lesions } \\
\text { FI: } 52 \\
\text { Constipation } 57\end{array}$ & $\begin{array}{l}\text { FU: nm } \\
\text { TT: nm }\end{array}$ & Not defined & $\begin{array}{l}\text { Single center } \\
\text { Prospective-controlled trial } \\
\text { Questionnaire evacuation habits } \\
10 \text { biofeedback sessions } \\
12 \text { conventional treatment (laxa- } \\
\text { tives, stimulants and enema) } \\
30 \text { no treatment }\end{array}$ & $\begin{array}{l}\text { Biofeedback: 2/10 improved, 4/10 } \\
\text { full bowel control } \\
\text { Conventional treatment: 7/12 } \\
\text { improved. } \\
\text { No statistical analysis done }\end{array}$ & Not evaluated \\
\hline $\begin{array}{l}\text { Shoshan } \\
\text { et al. }{ }^{15}\end{array}$ & $\begin{array}{l}20 \text { SB } \\
\text { Age: } 4-29 \text { years } \\
\text { LL: T4-L5 } \\
\text { FI: } 17 \text { diapers, } 3 \text { pads } \\
\text { Constipation: nm }\end{array}$ & $\begin{array}{l}\text { FU: } 5 \mathrm{w} \\
\mathrm{TT}: \mathrm{nm}\end{array}$ & Not defined & $\begin{array}{l}\text { Single center } \\
\text { Self-controlled clinical trial. } \\
\text { Daily record. } \\
\text { FU visits at week } 0,1,2 \text { and } 5 \text {. } \\
\text { Anal plug use start at week } 1 \text {. } \\
\text { Effect on FI scale } 0-4 \text { ( } 0=\text { not } \\
\text { bothersome, } 4=\text { very bothersome). }\end{array}$ & $\begin{array}{l}15 / 20 \text { completed the study } \\
\text { Accidents per week start: } 4(0-28) \text {, } \\
5 \text { w } 0(0-8)(P=.002)\end{array}$ & $\begin{array}{l}\text { Baseline } 50 \% \mathrm{Fl} \text { severely } \\
\text { impedes daily life. } \\
5 \text { w } 40 \% \text { slight interference } \\
\text { Significant reduction of } \mathrm{FI} \\
\text { scale }(P=0.001) \text {. }\end{array}$ \\
\hline $\begin{array}{l}\text { Malone } \\
\text { et al. }\end{array}$ & $\begin{array}{l}n=109 \mathrm{SB} \\
\text { Age: } 9-47.8 \text { years } \\
68 \text { wheelchair } \\
\text { LL: } n \mathrm{~m} \\
\mathrm{FI}: 55 \\
\text { Constipation: } \mathrm{nm}\end{array}$ & $\begin{array}{l}\text { FU: nm } \\
\text { TT: nm }\end{array}$ & Not defined & $\begin{array}{l}\text { Multicenter } \\
\text { Questionnaires } \\
\text { Random cohort }<2000 \text { patient } \\
\text { database } \\
\text { 109/144 responded }\end{array}$ & $\begin{array}{l}\text { 94/104 regular toileting } \\
\text { 26/104 manual evacuation } \\
\text { 25/104 laxatives } \\
\text { 13/103 suppositories } \\
55 / 104 \text { (53\%) regular soiled }\end{array}$ & Not evaluated \\
\hline Krogh et al. ${ }^{4}$ & $\begin{array}{l}n=125 \mathrm{SB} \\
\text { Age: } 2-18 \text { years } \\
\text { LL: } \mathrm{nm} \\
\mathrm{FI}: 55 \\
\text { Constipation: } \mathrm{nm}\end{array}$ & $\begin{array}{l}\text { FU: nm } \\
\text { TT: RCE: 133' } \\
\text { Digital: } 61 \text { times } \\
\text { No method 132' }\end{array}$ & $\begin{array}{l}\text { Stool loss < once } \\
\text { month }\end{array}$ & $\begin{array}{l}\text { Multicenter } \\
184 \text { item questionnaire (tested for } \\
\text { reproducibility and validity) and } \\
\text { validated } C B C L \\
125 / 208(100>4 \text { years old) } \\
\text { responded }\end{array}$ & $\begin{array}{l}\text { 25/125 digital evacuation } \\
\text { 13/125 suppositories } \\
\text { 35/125 RCE } \\
\text { 35/125 laxatives } \\
\text { 55/100 (children }>4 \text { years } \\
\text { old)(55\%) FI }\end{array}$ & $\begin{array}{l}\text { 10/42 FI major influence on } \\
\text { QoL in 2-5-year-olds } \\
21 / 46 \mathrm{FI} \text { major influence on } \\
\text { QoL in 6-10-year olds } \\
17 / 37 \mathrm{FI} \text { major influence on } \\
\text { QoL in 11-18-year-olds }\end{array}$ \\
\hline $\begin{array}{l}\text { Verhoef } \\
\text { et al. }{ }^{6}\end{array}$ & $\begin{array}{l}n=350 \text { SB } \\
\text { Age: } 16-25 \text { years } \\
70 \text { wheelchair } \\
\text { LL: L5-S1 lesions } \\
\text { FI: nm } \\
\text { Constipation: nm }\end{array}$ & $\begin{array}{l}\text { FU: } \mathrm{nm} \\
\text { TT: } 58 / \\
179>15 \mathrm{~min} \text { a } \\
\text { day }\end{array}$ & $\begin{array}{l}\text { Stool loss < once } \\
\text { per month }\end{array}$ & $\begin{array}{l}\text { Multicenter } \\
\text { Data collected from interviews and } \\
\text { neurophysiological testing, retro- } \\
\text { spective medical history } \\
179 \text { responded from } 350\end{array}$ & $\begin{array}{l}\text { 31/179 laxatives } \\
\text { 49/179 RCE } \\
\text { 27/179 manual evacuation } \\
61 / 179(34 \%) \mathrm{FI}\end{array}$ & $\begin{array}{l}47 / 61(77 \%) \text { perceived } \mathrm{FI} \text { as } \\
\text { a problem }\end{array}$ \\
\hline $\begin{array}{l}\text { Vande Velde } \\
\text { et al. }{ }^{5}\end{array}$ & $\begin{array}{l}n=80 \mathrm{SB} \\
\text { Age: } 5-18 \text { years } \\
33 \text { wheelchair } \\
\text { LL: } 26<\mathrm{S} 2,22 \text { L5-S1, } \\
32>\mathrm{L} 4 \\
\mathrm{FI}: \mathrm{nm} \\
\text { Constipation: } \mathrm{nm}\end{array}$ & $\begin{array}{l}\text { FU: } \mathrm{nm} \\
\text { TT: RCE and ACE } \\
21 \text { min a day } \\
\text { (8.5-35 min per } \\
\text { day) }\end{array}$ & $\begin{array}{l}\text { Stool loss }< \\
\text { once per week }\end{array}$ & $\begin{array}{l}\text { Single center } \\
\text { Descriptive cohort study } \\
\text { Stepwise therapeutic strategy }\end{array}$ & $\begin{array}{l}5 / 80 \text { regular toileting (no } \mathrm{FI}) \\
13 / 80 \text { manual evacuation ( } 38 \% \\
\mathrm{FI}) \\
24 / 80 \mathrm{RCE}(13 \% \mathrm{FI}) \\
16 / 80 \mathrm{ACE}(\mathrm{no} \mathrm{FI}) \\
22 / 80(27 \%) \mathrm{FI}\end{array}$ & Not evaluated \\
\hline $\begin{array}{l}\text { Shandling } \\
\text { and } \\
\text { Gilmour, }^{10}\end{array}$ & $\begin{array}{l}n=112 \mathrm{SB} \\
\text { Age: } 4-20 \text { years } \\
\text { LL: } \mathrm{nm} \\
\mathrm{FI}: 112 \\
\text { Constipation: } \mathrm{nm}\end{array}$ & $\begin{array}{l}\text { FU: } \mathrm{nm} \\
\text { TT: } 15-20 \mathrm{~min}\end{array}$ & $\begin{array}{l}\text { Stool loss }<4 \\
\text { times per month }\end{array}$ & $\begin{array}{l}\text { Single center } \\
\text { RCE with balloon catheter with } \\
\text { saline water } 20 \mathrm{ml} \mathrm{kg}^{-1} \text { every } 24- \\
48 \mathrm{~h}\end{array}$ & $\begin{array}{l}4 \text { dropped out } \\
5 \text { returned to RCE after initially } \\
\text { dropping out } \\
100 \% \text { continence rate }\end{array}$ & Not evaluated \\
\hline
\end{tabular}


Table 1 (Continued)

\begin{tabular}{|c|c|c|c|c|c|c|}
\hline Author & Sample size, age, type & FU/TT & Fl definition & Study design & Results - effectiveness & Results—satisfaction \\
\hline $\begin{array}{l}\text { Liptak and } \\
\text { Revell }{ }^{16}\end{array}$ & $\begin{array}{l}n=31,30 \mathrm{SB} \\
\text { Age: } 3-19 \text { years } \\
\text { LL: T-S4 } \\
\mathrm{FI}: 18 / 25 \\
\text { Constipation: } 14 / 25\end{array}$ & $\begin{array}{l}\text { FU: } 30 \text { months } \\
\text { TT: } 21 \mathrm{~min}\end{array}$ & No stool loss & $\begin{array}{l}\text { Single center } \\
\text { Prospective clinical trial. } \\
\text { After bowel cleaning, start RCE } \\
\text { with balloon catheter, every } 24- \\
48 \mathrm{~h} \text { with saline water } 20 \mathrm{ml} \mathrm{kg}^{-1} \text {. } \\
\text { Standardized questionnaire over } \\
\text { telephone or by visit. Satisfaction } \\
\text { rate } 1-4 \text { ( } 1=\text { extremely dissatis- } \\
\text { fied, } 4=\text { extremely satisfied). }\end{array}$ & $\begin{array}{l}6 \text { dropped out first } 3 \text { weeks } \\
9 \text { dropped out after } 18 \text { months } \\
\text { FI dropped from } 72(18 / 25) \text { to } \\
29 \%(7 / 25) \text { at } 18 \text { months and to } \\
6 \%(1 / 16) \text { after } 30 \text { months } \\
(P<0.01)\end{array}$ & $\begin{array}{l}\text { Mean satisfaction score } \\
\text { increased from } 1.1-2.8 \text { after } \\
18 \text { months and } 3.3 \text { after } \\
\text { 30months }(P<0.01)\end{array}$ \\
\hline $\begin{array}{l}\text { Schöller- } \\
\text { Gyüre } \\
\text { et al. }{ }^{17}\end{array}$ & $\begin{array}{l}n=53 \mathrm{SB} \\
\text { Age: } 7 \text { months-22 years } \\
\text { LL: nm } \\
\mathrm{FI}: 14 \\
\text { Constipation: } 14\end{array}$ & $\begin{array}{l}\text { FU: } 33 \text { months } \\
\text { TT: nm }\end{array}$ & No stool loss & $\begin{array}{l}\text { Single center } \\
\text { Case review and questionnaire. } \\
\text { RCE with cone catheter with tap } \\
\text { water } 20 \mathrm{ml} \mathrm{kg}^{-1} \text { every } 24 \mathrm{~h} \text {. } \\
\text { Frequent telephone contact. }\end{array}$ & $\begin{array}{l}41 \text { returned questionnaire } \\
27 / 41(66 \%) \text { complete fecal con- } \\
\text { tinence. } 6 / 41 \text { RCE painful, 3/41 } \\
\text { RCE unpleasant }\end{array}$ & $\begin{array}{l}\text { Parental satisfaction high in } \\
63 \% \text {, good in } 37 \% \text {. } \\
\text { Major disadvantage is time and } \\
\text { energy to perform RCE ( } 51 \%) \text {, } \\
\text { daily burden on family (39\%) }\end{array}$ \\
\hline Eire et al. ${ }^{18}$ & $\begin{array}{l}n=33 \mathrm{SB} \\
\text { Age: } 5-22 \text { years } \\
\text { LL: } n \mathrm{~m} \\
\mathrm{Fl}: \mathrm{nm} \\
\text { Constipation: } \mathrm{nm}\end{array}$ & $\begin{array}{l}\text { FU: } 30 \text { months } \\
\text { TT: Median } \\
30 \text { min (15- } \\
45 \text { min) }\end{array}$ & No stool loss & $\begin{array}{l}\text { Single center } \\
\text { Selected and well-motivated } \\
\text { patients } \\
\text { Retrospective case review. } \\
\text { After desimpaction, start RCE with } \\
\text { balloon catheter saline water, } \\
\text { median } 500 \mathrm{ml} \text { every } 24 \mathrm{~h} \text { and at } \\
\text { continence, every } 48 \mathrm{~h}\end{array}$ & $\begin{array}{l}\text { 32/33 became continent } \\
2 / 33 \text { were independent }\end{array}$ & Not evaluated \\
\hline $\begin{array}{l}\text { Mattsson } \\
\text { and Gladh }{ }^{19}\end{array}$ & $\begin{array}{l}n=40 \mathrm{SB} \\
\text { Age: } 10 \text { months- } 11 \text { years } \\
\text { LL: } n \mathrm{~m} \\
\mathrm{FI}: 40 \text { Constipation: } 40\end{array}$ & $\begin{array}{l}\text { FU: } 4 \text { months, } \\
\text { up to } 8 \text { years } \\
\text { after RCE } \\
\text { TT: } 12-60 \text { min }\end{array}$ & No stool loss & $\begin{array}{l}\text { Single center } \\
\text { Parental questionnaire (eight } \\
\text { questions). } \\
\text { RCE with cone catheter with tap } \\
\text { water, median } 300 \mathrm{ml} \text { every } 24 \mathrm{~h} \\
\text { Plasma sodium levels in } 28 \mathrm{SB} \\
\text { before start and after } 1 \text { month or } 1 \\
\text { year } \\
\text { Manometry in } 28 \mathrm{SB} \text { before and } \\
\text { after 1-3 years }\end{array}$ & $\begin{array}{l}5 \text { dropped out } \\
35 / 40 \text { were continent } \\
\text { All free of constipation } \\
\text { 1/40 was independent }\end{array}$ & $\begin{array}{l}35 / 40 \text { found RCE satisfactory } \\
\text { All found RCE time consum- } \\
\text { ing } \\
36 / 40 \text { parents reported gen- } \\
\text { eral improvement in well- } \\
\text { being }\end{array}$ \\
\hline $\begin{array}{l}\text { Ausili } \\
\text { et } a l^{20}\end{array}$ & $\begin{array}{l}n=60 \mathrm{SB} \\
\text { Age: } 8-17 \text { years } \\
\text { LL: } n \mathrm{~m} \\
\text { FI: } 16 \text { Constipation: } 60\end{array}$ & $\begin{array}{l}\text { FU: } 3 \text { months } \\
\text { TT: nm }\end{array}$ & $\begin{array}{l}\text { Stool loss }< \\
\text { once per month }\end{array}$ & $\begin{array}{l}\text { Single center } \\
\text { Prospective clinical trial. } \\
\text { Validated questionnaire (NBD } \\
\text { score, range } 0-47(47=\text { severe } \\
\text { bowel dysfunction) }{ }^{45} \text { ) and QoL } \\
\text { Visit at start and after } 3 \text { months } \\
\text { RCE with balloon catheter }\end{array}$ & $\begin{array}{l}36 / 60(60 \%) \text { relief of constipation } \\
12 / 16(75 \%) \text { relief of fecal } \\
\text { incontinence } \\
\text { NBD decreased from } 17.5 \text { to } 8.5 \\
\text { after treatment }(P<0.001)\end{array}$ & $\begin{array}{l}\text { Parents reported an improve- } \\
\text { ment on } Q \mathrm{oL} \text { and degree of } \\
\text { satisfaction } \\
\text { NBD score improved from } \\
17.5 \text { to } 8.5(P<0.001)\end{array}$ \\
\hline $\begin{array}{l}\text { Pereira } \\
\text { et } a l^{21}\end{array}$ & $\begin{array}{l}n=40,28 \mathrm{SB} \\
\text { Age: } 6-25 \text { years } \\
\text { LL: } n \mathrm{~m} \\
\mathrm{FI}: \mathrm{nm} \\
\text { Constipation: } \mathrm{nm}\end{array}$ & $\begin{array}{l}\text { FU: } 12 \text { months } \\
\text { TT: average } \\
\text { 15-30 min }\end{array}$ & $\begin{array}{l}\text { No stool loss } \\
\text { Pseudo-conti- } \\
\text { nence: no stool } \\
\text { loss with treat- } \\
\text { ment }\end{array}$ & $\begin{array}{l}\text { Single center } \\
\text { Prospective clinical trial } \\
\text { Standard questionnaire on bowel } \\
\text { function and QoL (rate } 0-10,0= \\
\text { great reduction, } 10=\text { great } \\
\text { improvement) } \\
\text { RCE with balloon catheter with } \\
\text { average } 616 \mathrm{ml} \text { tap water, every } 3 \\
\text { days }\end{array}$ & $\begin{array}{l}35 \text { returned questionnaire } \\
\text { Pseudo-continence rose from } 10 \text { / } \\
35 \text { to } 28 / 35 \text { ( } 80 \% \text { ) } \\
16 / 35 \text { partially or totally indepen- } \\
\text { dent } \\
\text { Significantly less time spent than } \\
\text { conventional bowel management }\end{array}$ & $\begin{array}{l}\text { Mean grade of satisfaction } \\
\text { was } 7.3\end{array}$ \\
\hline Choi et al. ${ }^{22}$ & $\begin{array}{l}n=53 \mathrm{SB} \\
\text { Age: } 3-13.8 \text { years } \\
\text { LL: } \mathrm{nm} \\
\mathrm{FI}: \mathrm{nm} \\
\text { Constipation: } \mathrm{nm}\end{array}$ & $\begin{array}{l}\text { FU: } 4 \text { months } \\
\text { TT: } 15.9 \text { min }\end{array}$ & $\begin{array}{l}\text { Stool loss <once } \\
\text { per week }\end{array}$ & $\begin{array}{l}\text { Single center } \\
\text { Prospective clinical trial } \\
\text { Survey questionnaire on bowel } \\
\text { symptoms, QoL and general char- } \\
\text { acteristics ( } 40 \text { items) } \\
\text { Stepwise bowel program: first } \\
\text { polyethylene glycol } 3350 \text { at } \\
0.5 \mathrm{~g} \mathrm{~kg}^{-1} \text { per day, if failure start } \\
\mathrm{RCE} \text { with cone or balloon catheter } \\
\text { with tap water every } 48-72 \mathrm{~h}\end{array}$ & $\begin{array}{l}6 / 53(11 \%) \text { success } \\
43 / 47(81 \%) \text { success } \\
\text { Bowel care time decreased from } \\
27 \text { to } 15.9 \text { min }(P=0.003) \\
\mathrm{FI} \text { per week decreased from } 6.9 \text { to } \\
0.5 \text { defecations per week } \\
(P=0.004)\end{array}$ & $\begin{array}{l}\text { Significant reduction in } \\
\text { impact on travel and sociali- } \\
\text { zation }(P=0.006) \\
\text { Significant reduction in care- } \\
\text { giver support and emotional } \\
\text { impact }(P<0.001)\end{array}$ \\
\hline
\end{tabular}

Abbreviations: ACE, antegrade colon enema; CBCL, child behavior checklist; FI, fecal incontinence; FU, follow-up; NBD, neurogenic bowel dysfunction; LL, lesion level; QoL, quality of life; RCE, retrograde colon enema; SB, spina bifida; TT, therapy time. 
emotional impact. ${ }^{22}$ However, two studies reported RCE to be 'a daily burden'. In both studies, the time spent and the energy needed to perform RCE were perceived as a major obstacle. ${ }^{17,19}$ Noncompliance and non-motivation could be a result of dissatisfaction with the treatment choice. King et al. ${ }^{12}$ reported a non-compliance rate of $28 \%$.

\section{Electrical stimulation}

The studies on electrical stimulation are summarized in Table 2.

Due to different stimulation techniques and result reports, the studies are not comparable. Most studies (3/4) used the above stated definition of pseudo-continence. ${ }^{23-25}$ Only the study of Kajbafzadeh et al. $^{26}$ used a less-strict pseudo-continence definition. The achieved pseudo-continence rate ranged from $50^{24}$ to $70 \% .{ }^{25}$ An increased spontaneous stool frequency was reported by two studies. ${ }^{23,26}$ Only one study reported a $73 \%$ decrease of constipation. The study of Marshall and Boston ${ }^{23}$ was the only double-blind randomized placebo-controlled trial reporting an important placebo effect (no actual data available).

\section{Non-conservative, surgical bowel management}

The studies are summarized in Table 3. During a surgical procedure, the appendix (or ileum if appendix is not available) is used to create a catheterizable stoma. Besides using the appendix or creating a stoma a cecostomy can be made laparoscopically or percutaneously. A stoma or cecostomy can be placed both right- or left-sided. In this review, mainly results on right-sided stoma were found.

Most studies (10/17) on surgical bowel management or ACE used the strict definition for fecal pseudo-continence (no stool loss or stool loss less than once a month). ${ }^{28,30-32,35-39,43}$ Some studies (5/17) gave no definition for fecal pseudo-continence ${ }^{27,29,33,40,41}$ or a less-strict definition. ${ }^{42}$ One study evaluated incontinence with a 5-point Likert scale. ${ }^{34}$ The reports on achieving fecal pseudo-continence using nonconservative bowel management varied from 60 (only adults) ${ }^{43}$ to 94\%. ${ }^{35,36}$ Again only the studies using the above-mentioned strict definition of pseudo-continence were used to calculate the overall effectiveness of non-conservative treatment. Fecal pseudo-continence was achieved in $81 \%$ (378/469). No study reported on constipation. The washout fluid used was saline in $4,{ }^{27,32,37,42}$ tap water in another $4^{31,35,36,39}$ and a combination of both in 2 studies. ${ }^{34,43}$ Five studies did

Table 2 Summary of papers on electrical stimulation in SB patients

\begin{tabular}{|c|c|c|c|c|c|c|}
\hline Author & Sample & $\begin{array}{l}\text { FU/defecation } \\
\text { time }\end{array}$ & Definition FI & Study design & Findings - effectiveness & Findings-satisfaction \\
\hline $\begin{array}{l}\text { Marshall } \\
\text { and } \\
\text { Boston } 23\end{array}$ & $\begin{array}{l}n=50 \text { SB } \\
\text { Age: } 4-18 \text { years } \\
\text { LL: } n m \\
\text { FI: nm } \\
\text { Constipation: nm }\end{array}$ & $\begin{array}{l}\text { FU: } 6 \text { weeks } \\
\text { TT: } \mathrm{nm}\end{array}$ & Not defined & $\begin{array}{l}\text { Single center } \\
\text { Randomized double-blind pla- } \\
\text { cebo-controlled trial } \\
\text { Daily } 1 \text { h at home ES stimula- } \\
\text { tion on skin: } 26 \text { treatment, } 24 \\
\text { sham } \\
\text { Regular telephone contact to } \\
\text { stimulate compliance } \\
1 \text { week diary }\end{array}$ & $\begin{array}{l}49 \% \text { more spontaneous stools } \\
\text { in active ES but not significant } \\
\text { Important placebo effect } \\
\text { No adverse effects }\end{array}$ & Not evaluated \\
\hline $\begin{array}{l}\text { Palmer } \\
\text { et al. }{ }^{24}\end{array}$ & $\begin{array}{l}n=55 \text { SB } \\
\text { Age: } 2-14 \text { years } \\
\text { LL: } n m \\
\text { FI: nm } \\
\text { Constipation: nm }\end{array}$ & $\begin{array}{l}\text { FU: } 12-72 \\
\text { months } \\
\text { TT: nm }\end{array}$ & $\begin{array}{l}\text { Improvement: } \\
\text { - less defecations } \\
\text { - better rectal } \\
\text { sensation } \\
\text { - ability to hold } \\
\text { consciously }\end{array}$ & $\begin{array}{l}\text { Single center } \\
\text { Prospective clinical trial } \\
\text { Home transrectal ES } \\
30 \text { min per day, } 5 \text { days per week }\end{array}$ & $\begin{array}{l}20 / 55 \text { (36\%) complete success } \\
\text { (improvement of all parameters) } \\
30 / 55 \text { (55\%) moderate success } \\
\text { (improvement of any parameter) } \\
71 \% \text { complete bowel conti- } \\
\text { nence } \\
\text { No adverse effects }\end{array}$ & $\begin{array}{l}\text { Inclusion of parental sub- } \\
\text { jective opinion decreased } \\
\text { the success rate of this } \\
\text { therapy }\end{array}$ \\
\hline Han et al. ${ }^{25}$ & $\begin{array}{l}n=24 \text { SB } \\
\text { Age: } 4-13 \text { years } \\
\text { LL: } n m \\
\text { FI: nm } \\
\text { Constipation: nm }\end{array}$ & $\begin{array}{l}\text { FU: } 15.8 \\
\text { months } \\
\text { TT: nm }\end{array}$ & No stool loss & $\begin{array}{l}\text { Single center } \\
\text { Retrospective case review } \\
\text { Daily } 1 \text { h at home transurethral } \\
\text { ES, } 5 \text { days per week for } 4 \text { weeks } \\
\text { FU cycles: } 2 \text { weeks every 3-6 } \\
\text { months } \\
\text { One week diary before treatment } \\
\text { and subsequent cycles }\end{array}$ & $\begin{array}{l}\text { Mean episodes of fecal incon- } \\
\text { tinence decreased significantly } \\
\text { from } 7.3-4.8 \text { a week }(P<0.05) \\
\text { Complete fecal continence in } \\
50 \%(12 / 24) \\
\text { Urinary tract infections in } 10 \\
\text { patients during transurethral ES }\end{array}$ & Not evaluated \\
\hline $\begin{array}{l}\text { Kajbafza- } \\
\text { deh et al. }{ }^{26}\end{array}$ & $\begin{array}{l}n=30 \text { SB } \\
\text { Age: } 3-12 \text { years } \\
\text { LL: } n m \\
\text { FI: nm } \\
\text { Constipation: nm }\end{array}$ & $\begin{array}{l}\text { FU: nm } \\
\text { TT: nm }\end{array}$ & $\begin{array}{l}\text { Stool loss }<\text { once } \\
\text { per week }\end{array}$ & $\begin{array}{l}\text { Single center } \\
\text { Randomized controlled trial } \\
\text { Home cutaneous ES for } 20 \text { min, } \\
3 \text { times per week: } 15 \text { treatment, } \\
15 \text { sham } \\
\text { Bowel habit diary, NBD: range } \\
0-47 \text { ( } 47=\text { severe bowel dys- } \\
\text { function) } 45 \\
\text { Manometry before and } \\
6 \text { months after treatment }\end{array}$ & $\begin{array}{l}\text { Constipation is decreased in } 11 \text { / } \\
15(73 \%) \text { and remained in } 8 / 15 \\
\text { ( } 53 \%) \text { after } 6 \text { months } \\
\text { Significant increase of stool } \\
\text { frequency from } 2.5 \text { to } 4.7 \text { stools } \\
\text { per week after treatment } \\
\text { Significant improvement mano- } \\
\text { metry measures } \\
\text { No adverse effects }\end{array}$ & Not evaluated \\
\hline
\end{tabular}


Table 3 Summary of papers on non-conservative bowel management in SB patients

\begin{tabular}{|c|c|c|c|c|c|c|}
\hline Author & Sample & $\begin{array}{l}\text { FU/ defecation } \\
\text { time }\end{array}$ & $\begin{array}{l}\text { Definition fecal } \\
\text { continence }\end{array}$ & Study design & $\begin{array}{l}\text { Findings_effectiveness } \\
\text { Findings_complications }\end{array}$ & Findings-satisfaction \\
\hline $\begin{array}{l}\text { Hensle } \\
\text { et al. } 27\end{array}$ & $\begin{array}{l}n=27 \mathrm{SB} \\
\text { Age: } 10-31 \text { years } \\
22 \text { wheelchair } \\
\text { LL: } \mathrm{nm} \\
\mathrm{FI}: \mathrm{nm} \\
\text { Constipation: } \mathrm{nm}\end{array}$ & $\begin{array}{l}\text { FU: } 9-30 \\
\text { months } \\
\text { TT: } 30 \mathrm{~min}\end{array}$ & Not defined & $\begin{array}{l}\text { Single center } \\
\text { Retrospective case review } \\
\text { Malone ACE, eight concomitant } \\
\text { urologic surgery } \\
\text { Saline water } 300 \mathrm{ml} \\
\text { Standard QoL questionnaire }\end{array}$ & $\begin{array}{l}\text { Two dropped out } \\
\text { 19/25 }(76 \%) \text { : complete bowel } \\
\text { control } \\
\text { 10/27 complications } \\
3 / 27 \text { redo surgery }\end{array}$ & $\begin{array}{l}25 / 27 \text { reported substantial } \\
\text { improvement in their QoL }\end{array}$ \\
\hline $\begin{array}{l}\text { Shankar } \\
\text { et al. }{ }^{28}\end{array}$ & $\begin{array}{l}n=40,27 \mathrm{SB} \\
\text { Age: } 6-21 \text { years } \\
14 \text { wheelchair } \\
\text { LL: nm } \\
\text { FI: } n m \\
\text { Constipation: nm }\end{array}$ & $\begin{array}{l}\text { FU: } 21 \text { months } \\
\text { TT: } n m\end{array}$ & $\begin{array}{l}\text { Stool loss }< \\
\text { once per month }\end{array}$ & $\begin{array}{l}\text { Single center } \\
\text { Retrospective case review } \\
\text { Malone ACE every } 48 \mathrm{~h} \\
\text { QOLI score: 0-5 (5: ideal) } \\
\text { CTT and manometry in } 34\end{array}$ & $\begin{array}{l}4 \text { dropped out } \\
17 / 27(63 \%) \text { success } \\
23 / 40 \text { complications } \\
11 / 40 \text { redo surgery }\end{array}$ & $\begin{array}{l}\text { Mean QOLI: 3.5, all report } \\
\text { some improvement in QoL } \\
\text { QOLI significantly lower in } \\
\text { SB wheelchair group } \\
(P=0.033)\end{array}$ \\
\hline $\begin{array}{l}\text { Webb } \\
\text { et al. }{ }^{29}\end{array}$ & $\begin{array}{l}n=57,43 \mathrm{SB} \\
\text { Age: } 5-30 \text { years } \\
\text { LL: } n \mathrm{~m} \\
\mathrm{FI}: \mathrm{nm} \\
\text { Constipation: } \mathrm{nm}\end{array}$ & FU: 30 months & Not defined & $\begin{array}{l}\text { Single center } \\
\text { Retrospective case review } \\
\text { Malone ACE every } 72 \mathrm{~h} \text { phos- } \\
\text { phosoda or soapy water, } 21 \\
\text { concomitant urologic surgery }\end{array}$ & $\begin{array}{l}2 \text { dropped out } \\
\text { 40/43: good to excellent results } \\
8 / 57 \text { complications } \\
3 / 57 \text { redo surgery }\end{array}$ & $\begin{array}{l}\text { Overall patient satisfaction } \\
\text { is good to excellent }\end{array}$ \\
\hline $\begin{array}{l}\text { Curry } \\
\text { et al. }{ }^{30}\end{array}$ & $\begin{array}{l}n=273,108 \mathrm{SB} \\
\text { Age: } 7.5-29.9 \text { years } \\
\text { LL: } n \mathrm{~m} \\
\mathrm{FI}: \mathrm{nm} \\
\text { Constipation: } \mathrm{nm}\end{array}$ & 30 months & $\begin{array}{l}\text { Full: totally } \\
\text { clean or minor } \\
\text { leakage on } \\
\text { night of } \\
\text { washout }\end{array}$ & $\begin{array}{l}\text { Multicenter retrospective pro- } \\
\text { forma } \\
\text { Malone ACE or button (19/273) }\end{array}$ & $\begin{array}{l}68 / 108(63 \%) \text { full success } \\
111 / 27 \text { three complications }\end{array}$ & Not evaluated \\
\hline $\begin{array}{l}\text { Aksnes } \\
\text { et al. }{ }^{31}\end{array}$ & $\begin{array}{l}n=20 \mathrm{SB} \\
\text { Age: } 6.3-17 \text { years } \\
\text { LL: } n \mathrm{~m} \\
\mathrm{FI}: 16 \text { Constipation: } 4\end{array}$ & $\begin{array}{l}\text { FU: } 16 \text { months } \\
\text { TT: } 50 \text { min } \\
(30-75)\end{array}$ & No stool loss & $\begin{array}{l}\text { Single center } \\
\text { Prospective clinical trial } \\
\text { Malone ACE } 900 \mathrm{ml} \text { tap water } \\
\text { Standardized questionnaire } \\
\text { before and } 6 \text { months after ACE, } \\
\text { CBCL, YSR, SPPA } 1-4 \text { score } \\
(4=\text { describes me very well) } \\
\text { FU visit and telephone }\end{array}$ & $\begin{array}{l}\text { 16/20 gained independency } \\
16 / 20 \text { complete continence } \\
6 / 20 \text { redo surgery }\end{array}$ & $\begin{array}{l}\text { SPPA: postoperative } \\
\text { improved self-esteem } \\
(P=.04) \text { and close friends } \\
(P=.006) \\
\text { CBCL and YSR no differ- } \\
\text { ence pre- and postoperative }\end{array}$ \\
\hline Dey et al. ${ }^{32}$ & $\begin{array}{l}n=62,31 \mathrm{SB} \\
\text { Age: } 3.8-21.4 \text { years } \\
\text { LL: } n \mathrm{~m} \\
\mathrm{FI}: \mathrm{nm} \\
\text { Constipation: } \mathrm{nm}\end{array}$ & $\begin{array}{l}\text { FU: } 65 \text { months } \\
\text { TT: } 53 \text { min } \\
(15-180)\end{array}$ & $\begin{array}{l}\text { Stool loss }< \\
\text { once per month }\end{array}$ & $\begin{array}{l}\text { Single center } \\
\text { Retrospective casenote review } \\
\text { Appendix ACE } 550 \mathrm{ml} \text { saline } \\
\text { water } \\
\text { Questionnaire: } 32 \text { responded } \\
\text { from } 51 \text { still using ACE }\end{array}$ & $\begin{array}{l}11 \text { dropped out } \\
27 / 32 \text { (84\%) continent } \\
35 / 62 \text { complications } \\
\text { 22/62 redo surgery }\end{array}$ & $\begin{array}{l}\text { Median satisfaction score: } 9 \\
\text { (10=completely satisfied) } \\
\text { Significant correlation } \\
\text { between continence and } \\
\text { satisfaction }(P=0.04)\end{array}$ \\
\hline $\begin{array}{l}\text { Casale } \\
\text { et al. }\end{array}$ & $\begin{array}{l}n=275 \mathrm{SB} \\
\text { Age: mean } 11 \text { years } \\
\text { LL: } n \mathrm{~m} \\
\mathrm{FI}: \mathrm{nm} \\
\text { Constipation: } \mathrm{nm}\end{array}$ & $\begin{array}{l}\text { FU: } 45-120 \\
\text { months } \\
\text { TT: nm }\end{array}$ & Not defined & $\begin{array}{l}\text { Single center } \\
\text { Retrospective chart review } \\
215 \text { Malone ACE } \\
\text { Single surgery compared with } \\
\text { combined urologic surgery } \\
(n=158)\end{array}$ & $\begin{array}{l}94 \% \text { continence, no difference } \\
\text { between groups } \\
27 \% \text { complications, no differ- } \\
\text { ence between groups }\end{array}$ & Not evaluated \\
\hline $\begin{array}{l}\text { Lemelle } \\
\text { et al. }{ }^{34}\end{array}$ & $\begin{array}{l}n=423 \text { SB } \\
\text { Age: } 10-47 \text { years } \\
145 \text { wheelchair } \\
\text { LL: } n m \\
\text { FI: } n m \\
\text { Constipation: } n m\end{array}$ & $\begin{array}{l}\text { FU: } 36 \text { months } \\
\text { TT: } 50 \text { min } \\
(15-90)\end{array}$ & $\begin{array}{l}\text { 5-point Likert } \\
\text { scale }(1= \\
\text { permanent, } \\
2=\text { frequent, } \\
3=\text { occasional, } \\
4=\text { rare, } \\
5=\text { never })\end{array}$ & $\begin{array}{l}\text { Multicentre } \\
\text { Clinical interview and medical } \\
\text { chart review } \\
\text { Right ACE: } 40 \text {, left ACE: } 7 \text {, } \\
1.2 \text { L tap or saline water } \\
\text { Conventional treated group } \\
\text { ( } n=382) \text { compared to ACE } \\
\text { group }(n=41)\end{array}$ & $\begin{array}{l}6 \text { dropped out } \\
\text { ACE group is significant younger } \\
\text { ACE group significant less FI } \\
\text { Left ACE tends to shorter defe- } \\
\text { cation time and worse FI }\end{array}$ & Not evaluated \\
\hline $\begin{array}{l}\text { Bani-Hani } \\
\text { et al. }\end{array}$ & $\begin{array}{l}n=236,199 \mathrm{SB} \\
\text { Age: } 2-36 \text { years } \\
\text { LL: } \mathrm{nm} \\
\mathrm{FI}: \mathrm{nm} \\
\text { Constipation: } \mathrm{nm}\end{array}$ & $\begin{array}{l}\text { FU: } 50 \text { months } \\
\text { TT: } n m\end{array}$ & No stool loss & $\begin{array}{l}\text { Single center } \\
\text { Retrospective chart review } \\
\text { Malone ACE daily } 642 \mathrm{ml} \text { tap } \\
\text { water } \\
\text { Evaluation of use of additives: } \\
\text { - immediate } \mathrm{FI} \text { : larger volume or } \\
\text { more time on toilet } \\
\text { - midday FI: bowel cleanout and } \\
\text { add } 17 \mathrm{mg} \text { MiraLAX }\end{array}$ & $\begin{array}{l}196 / 236(83 \%) \text { continence } \\
\text { rose to } 221 / 236(94 \%) \text { using } \\
\text { additives }\end{array}$ & Not evaluated \\
\hline
\end{tabular}


Table 3 (Continued)

\begin{tabular}{|c|c|c|c|c|c|c|}
\hline Author & Sample & $\begin{array}{l}\text { FU/ defecation } \\
\text { time }\end{array}$ & $\begin{array}{l}\text { Definition fecal } \\
\text { continence }\end{array}$ & Study design & $\begin{array}{l}\text { Findings - effectiveness } \\
\text { Findings—complications }\end{array}$ & Findings-satisfaction \\
\hline $\begin{array}{l}\text { Cain } \\
\text { et al. }\end{array}$ & $\begin{array}{l}n=236,199 \mathrm{SB} \\
\text { Age: } 2-36 \text { years } \\
\text { LL: } \mathrm{nm} \\
\mathrm{FI}: \mathrm{nm} \\
\text { Constipation: } \mathrm{nm}\end{array}$ & $\begin{array}{l}\text { FU: } 50 \text { months } \\
\text { TT: } n m\end{array}$ & No stool loss & $\begin{array}{l}\text { Single center } \\
\text { Retrospective chart review } \\
\text { Malone ACE daily, } 642 \mathrm{ml} \text { tap } \\
\text { water }\end{array}$ & $\begin{array}{l}\text { 221/236 (94\%) continent } \\
\text { 98/236 complications } \\
\text { 51/236 redo surgery }\end{array}$ & Not evaluated \\
\hline $\begin{array}{l}\text { Wong } \\
\text { et } a l^{37}\end{array}$ & $\begin{array}{l}n=64,41 \text { SB } \\
\text { Age: } 6-15 y \\
\text { LL: } n m \\
\text { FI: nm } \\
\text { Constipation: nm }\end{array}$ & $\begin{array}{l}\text { FU: } \mathrm{nm} \\
\mathrm{TT}: 5-60 \mathrm{~min}\end{array}$ & No stool loss & $\begin{array}{l}\text { Single center } \\
\text { Retrospective chart review } \\
\text { Interviews and telephone, } \\
\text { structured questionnaire score } \\
0-24 \text { ( } 0=\text { full continence, } \\
24=\text { complete incontinence) } \\
\text { Chait cecostomy glycerin or } \\
\text { saline water every 48-72 h }\end{array}$ & $\begin{array}{l}\text { FI score in SB improved from } \\
18 \text { to } 7 \\
28 / 64 \text { complications }\end{array}$ & Not evaluated \\
\hline $\begin{array}{l}\text { Yardley } \\
\text { et al. }\end{array}$ & $\begin{array}{l}n=61,27 \mathrm{SB} \\
\text { Age: } 15.5-35.1 \text { years } \\
\mathrm{LL}: \mathrm{nm} \\
\mathrm{FI}: \mathrm{nm} \\
\text { Constipation: } \mathrm{nm}\end{array}$ & $\begin{array}{l}\text { FU: } 132 \\
\text { months } \\
\text { TT: nm }\end{array}$ & $\begin{array}{l}\text { Stool loss }< \\
\text { once per month }\end{array}$ & $\begin{array}{l}\text { Single center } \\
\text { Postal/telephone questionnaire }\end{array}$ & $\begin{array}{l}25 \text { dropped out } \\
11 / 16(69 \%) \text { SB continent } \\
\text { 5/36 complications } \\
\text { 2/36 redo surgery }\end{array}$ & $\begin{array}{l}\text { Satisfaction score of ACE } \\
\text { users: } 4 \text { ( } 1=\text { very unsatis- } \\
\text { fied, } 5=\text { very satisfied) } \\
\text { High dissatisfaction with } \\
\text { transitional care }\end{array}$ \\
\hline $\begin{array}{l}\text { Matsuno } \\
\text { et al. }\end{array}$ & $\begin{array}{l}n=25 \mathrm{SB} \\
\text { Age: } 4.1-23.1 \text { years } \\
5 \text { wheelchair } \\
\text { LL: } \mathrm{nm} \\
\mathrm{FI}: \mathrm{nm} \\
\text { Constipation: } \mathrm{nm}\end{array}$ & $\begin{array}{l}\text { FU: } 27.5 \\
\text { months } \\
\text { TT: } 37.7 \mathrm{~min} \\
(20-60)\end{array}$ & No stool loss & $\begin{array}{l}\text { Single center } \\
\text { Comparing RCE to ACE retro- } \\
\text { spectively } \\
\text { - } 13 \text { patients RCE } \\
\text { - } 12 \text { patients ACE } \\
\text { tap water, daily to every } 48 \mathrm{~h} \\
\text { age is significantly }(P=0.009) \\
\text { younger in RCE compared with } \\
\text { ACE }\end{array}$ & $\begin{array}{l}\text { 10/13 (77\%) RCE pseudo-con- } \\
\text { tinent } \\
\text { 8/12 (75\%) ACE pseudo-conti- } \\
\text { nent } \\
\text { 3/13 RCE independent } \\
\text { 8/12 ACE independent }\end{array}$ & Not evaluated \\
\hline $\begin{array}{l}\text { Bar-Yosef } \\
\text { et al. }{ }^{40}\end{array}$ & $\begin{array}{l}n=21 \text { SB } \\
\text { Age: } 6-22 \text { years } \\
\text { LL: } n m \\
\text { FI: } n m \\
\text { Constipation: } n m\end{array}$ & $\begin{array}{l}\text { FU: } 56 \text { months } \\
\text { TT: } n m\end{array}$ & Not defined & $\begin{array}{l}\text { Single center } \\
\text { Retrospective chart review } \\
\text { Artificial urinary sphincter and } \\
\text { Malone ACE }\end{array}$ & $\begin{array}{l}19 / 21(90 \%) \text { full fecal conti- } \\
\text { nence } \\
3 / 21 \text { complications } \\
2 / 21 \text { redo surgery }\end{array}$ & Not evaluated \\
\hline $\begin{array}{l}\text { Ok and } \\
\text { Kurzrock }^{41}\end{array}$ & $\begin{array}{l}n=23 \text { SB families } \\
\text { Age: } n m \\
\text { LL: } n m \\
\text { FI: } n m \\
\text { Constipation: } n m\end{array}$ & $\begin{array}{l}\text { FU: } 6 \text { months } \\
\text { TT: } 45 \text { min }\end{array}$ & Not defined & $\begin{array}{l}\text { Single center } \\
\text { FICQoL: } 51 \text { item questionnaire } \\
\text { before and } 6 \text { months after ACE } \\
\text { procedure } \\
\text { - } 23 \text { families before surgery } \\
\text { - } 18 \text { families after surgery }\end{array}$ & $\begin{array}{l}\text { Diaper need decreased } \\
\text { Accident number improved from } \\
3.9 \text { to } 0.3 \text { per week }(P<0.01)\end{array}$ & $\begin{array}{l}\text { Significant improvement in } \\
\text { caretaker anxiety, depres- } \\
\text { sion and bother }\end{array}$ \\
\hline $\begin{array}{l}\text { Siddiqui } \\
\text { et al. }{ }^{42}\end{array}$ & $\begin{array}{l}n=105,40 \mathrm{SB} \\
\text { Age: median } 11.1 \text { years } \\
\text { LL: } n \mathrm{~m} \\
\mathrm{FI}: 21 \text { (10/40 SB) } \\
\text { Constipation: } 38 \\
\text { Mixed: } 46\end{array}$ & $\begin{array}{l}\text { FU: } 6 \text { months } \\
\text { TT: } \\
51.7 \pm 3.5 \mathrm{~min}\end{array}$ & $\begin{array}{l}\text { Stool loss }< \\
\text { once per week }\end{array}$ & $\begin{array}{l}\text { Single center } \\
\text { Retrospective chart review } \\
\text { Percutaneous or surgical ACE } \\
847 \mathrm{ml} \text {, GoLYTELY or saline } \\
\text { water every } 24-48 \mathrm{~h} \\
20 \text { concomitant urologic surgery }\end{array}$ & $\begin{array}{l}\text { 30/40 (75\%) SB continent } \\
74 / 117 \text { complications } \\
\text { 39/117 redo surgery } \\
\text { Significant increase in total } \\
\text { complications with poor out- } \\
\text { come in percutaneous ACE }\end{array}$ & Not evaluated \\
\hline $\begin{array}{l}\text { Vande } \\
\text { Velde } \\
\text { et al. }{ }^{43}\end{array}$ & $\begin{array}{l}n=40 \text { SB } \\
25 \text { children age: } \\
5-17 \text { years } \\
15 \text { adults age: } 18-38 \\
\text { years } \\
16 \text { wheelchair } \\
\text { LL: } 8 \mathrm{~S}, 30 \mathrm{~L}, 2 \mathrm{~T} \\
\mathrm{FI}: \mathrm{nm} \\
\text { Constipation: } \mathrm{nm}\end{array}$ & $\begin{array}{l}\text { Children } \\
\text { FU: } 60 \text { months } \\
\text { TT: } 35 \text { min } \\
\text { (15-60) } \\
\text { Adults } \\
\text { FU: } 132 \\
\text { months } \\
\text { TT: } 60 \text { min } \\
\text { (30-120) }\end{array}$ & No stool loss & $\begin{array}{l}\text { Single center } \\
\text { Retrospective cohort description } \\
\text { Questionnaire at clinic } \\
\text { Comparing RCE and ACE use in } \\
\text { children and adults } \\
\text { - child } 18 \text { RCE, 7ACE } \\
\text { - adult } 4 \text { RCE, } 11 \text { ACE }\end{array}$ & $\begin{array}{l}5 \text { dropped out } \\
19 / 25 \text { ( } 76 \%) \text { children continent } \\
\text { 9/15 (60\%) adults continent } \\
5 / 25 \text { children independent } \\
8 / 15 \text { adults independent } \\
\text { Significant relation between } \\
\text { follow-up and continence }\end{array}$ & $\begin{array}{l}2 / 6 \text { children perceived } \\
\text { incontinence as social pro- } \\
\text { blem compared with } 4 / 6 \\
\text { adults did }\end{array}$ \\
\hline
\end{tabular}

Abbreviations: ACE, antgrade continence enema; CBCL, child behavior checklist; CTT, colonic transit time; FICQoL, fecal incontinence and constipation QoL; LL, lesion level; QoL, quality of life; QOLI, QoL improvement; RCE, retrograde continence enema; SB, spina bifida; SPPA, self-perception profile for adolescents; TT, therapy time; YSR, youth self-report. 
not mention the type of fluid used. ${ }^{28,30,33,40,41}$ Most authors do not mention the volumes used (9/17). ${ }^{28-30,33,37-41}$ When mentioned, volumes varied between $300^{27}$ and $1500 \mathrm{ml}$ (in adults). ${ }^{43}$ The time spent for treatment was not reported in nine studies. ${ }^{28-30,33,35,36,38-40}$ If reported, it varied from $30^{27,37,43}$ to 50 min..$^{31,32,34,41-43}$

Complications are an important issue in case of surgical treatment. The complication rate varied widely. It was not clear whether studies reported all complications in a comparable way. The follow-up time of the different studies is different, also leading to a different complication rate. The most frequently reported complications were stomal stenosis, wound infections and perforations. Redo surgery for complications varied from $5^{29,38}$ over $10 \%{ }^{27,36,40}$ to an even higher rate of $30 \%{ }^{28,31,32,42}$ Overall redo surgery was necessary in $23 \%$ (142/616) of patients.

Comparison of the satisfaction rate was not attempted due to the differences in questionnaires and scales used. An improved satisfaction or a high satisfaction rate when using ACE was reported in six studies. ${ }^{27-29,31,32,38}$ One study described a significant improvement in anxiety, depression and bother of both caretakers and patients. ${ }^{41}$ However, most studies report a drop-out rate of $5^{29}$ to $30 \% .^{38}$ This could be perceived as dissatisfaction of the used strategy. Lack of transition care is, as reported by one study, also an important reason for dissatisfaction. This issue was also reflected in a difference of pseudo-continence rate between children and adults as described in one study, with children achieving higher rates associated with stricter follow-up. ${ }^{43}$

\section{DISCUSSION}

Most studies included in the review report on case series or patient cohorts without controls and therefore no conclusions are drawn regarding best mode of treatment. Further on, data were collected retrospectively in most studies and only a few used standardized questionnaires. Follow-up since starting treatment varied widely. Only two randomized trials both on electrical stimulation were identified. One was a double-blind placebo-controlled study, including, however, a very small patient group without clear end points. Therefore, stating recommendations or drawing conclusions on the different types of treatments used is tentative.

The results indicated that several treatment strategies can achieve pseudo-continence. Large colon washouts, retrograde or antegrade (surgical), however, seem to provide the best overall outcome. The RCE-treated patient group $(n=190)$ is smaller and has a shorter follow-up period compared with the patients treated by surgery. Despite the good pseudo-continence results, most authors state that surgery remains the final step in bowel management because of the amount of complications and drop-outs associated with this treatment modality. Some also consider ACE procedures in case of urological interventions. RCE, should be considered as a first-line treatment when both patients and parents are willing to invest in fecal pseudo-continence. Rigorous follow-up and problem solving in case of treatment failure with extra attention for transition care from adolescence to adulthood is of major importance in becoming and staying fecal pseudo-continent.

For all the treatment strategies used, the balance between successful therapy and the daily time investment will influence the compliance. Several studies tried to measure the impact of both fecal incontinence and treatment issues on social life and daily burden. The wide variety in disease severity as well as the multitude of health issues involved make the QoL measurement especially difficult.

Most centers use a stepwise and individually tailored protocol in the treatment of fecal incontinence with ACE surgery as a last step.
These programs are largely experience based and hardly evidence based. A common relevant definition of fecal pseudo-continence is needed to compare study results. In order to ameliorate results and gain insight into which treatment suits which patient, multicenter comparative trials will be needed in patients with comparable impairments, using standardized QoL outcome measurements. Cross-over trials are needed to compare the effect of different irrigation modalities in both RCE and ACE.

\section{CONCLUSION}

Irrigations both retrograde and antegrade are valuable treatment options in becoming fecal continent for SB patients. Surgery is currently used as final step in achievement of pseudo-continence. The burden of treatment can be important and should be accounted for. More research is needed to evaluate different techniques, long-term results of treatment and prediction of success or failure using clearly defined fecal pseudo-continence as a primary goal and standardized QoL evaluation as a secondary goal. Motivational support and strict follow-up of SB patients regarding fecal incontinence have an important role in the outcome.

\section{DATA ARCHIVING}

There were no data to deposit.

\section{CONFLICT OF INTEREST}

The authors declare no conflict of interest.

1 Vinck A, Nijhuis-van der Sanden MW, Roeleveld NJ, Mullaart RA, Rotteveel JJ, Maassen BA. Motor profile and cognitive functioning in children with spina bifida. Eur J Paediatr Neurol 2010; 14: 86-92.

2 Churchill BM, Abramson RP, Wahl EF. Dysfunction of the lower urinary and distal gastrointestinal tracts in pediatric patients with known spinal cord problems. Pediatr Clin North Am 2001; 48: 1587-1630.

3 Zickler CF, Richardson V. Achieving continence in children with neurogenic bowel and bladder. J Pediatr Health Care 2004; 18: 276-283.

4 Krogh K, Lie HR, Bilenberg N, Laurberg S. Bowel function in Danish children with myelomeningocele. APMIS 2003; 109: S81-S85.

5 Vande Velde S, Van Biervliet S, Van Renterghem K, Van Laecke E, Hoebeke P, Van Winckel M. Achieving fecal continence in patients with spina bifida: a descriptive cohort study. J Urol 2007; 178: 2640-2644; discussion 4

6 Verhoef M, Lurvink M, Barf HA, Post MW, Van Asbeck FW, Gooskens RH et al. High prevalence of incontinence among young adults with spina bifida: description, prediction and problem perception. Spinal Cord 2005; 43: 331-340.

7 Malone P, Wheeler R, Williams J. Continence in patients with spina bifida: long term results. Arch Dis Child 1994; 70: 107-110.

8 Lie HR, Lagergren J, Rasmussen F, Lagerkvist B, Hagelsteen J, Borjeson MC. Bowel and bladder control of children with myelomeningocele: a Nordic study. Dev Med Child Neurol 1991; 33: 1053-1061.

9 Centre for Reviews and Dissemination Systematic Reviews. CRD's Guidance For Undertaking Reviews in Healthcare. University of York: York, North Yorkshire, UK, 2008.

10 Shandling B, Gilmour RF. The enema continence catheter in spina bifida patients: successful bowel management. J Pediatr Surg 1987; 22: 271-273.

11 Malone PS, Ransley PG, Kiely EM. Preliminary report; the antegrade continence enema. Lancet 1990; 336: 1217-1218.

12 King JC, Currie DM, Wright E. Bowel training in spina bifida: importance of education, patients compliance, age and anal reflexes. Arch Phys Med Rehabil 1994; 75 243-247.

13 Whitehead WE, Parker L, Bosmajian L, Morrill-Corbin ED, Middaugh S, Garwood M et al. Treatment of fecal inctontinence in children with spina bifida: comparison of biofeedback and behaviour modification. Arch Phys Med Rehabil 1986; 67. 218-224

14 Ponticelli A, lacobelli BD, Silveri M, Broggi G, Rivosecchi M, De Gennaro M. Colorecta dysfunction and faecal incontinence in children with spina bifida. Br J Urol 1998; 81 (Suppl 3), 117-119.

15 Soshan L, Ben-Zvi D, Katz-Leurer M. Use of the anal plug in the treatment of fecal incontinence in patients with myelomeningocele. J Pediatr Nurs 2008; 23: 395-399.

16 Liptak GS, Revell GM. Management of bowel dysfunction in children with spinal cord disease or injury by means of the enema continence catheter. J Pediatr 1992; 120 190-194. 
17 Schöller-Gyüre M, Nesselaar C, van Wieringen H, van Gool JD. Treatment of defecation disorders by colonic enemas in children with spina bifida. Eur J Pediatr Surg 1996; 6 (Suppl I), 32-34.

18 Eire PF, Cives RV, Gago MC. Faecal incontinence in children with spina bifida: the best conservative treatment. Spinal Cord 2008; 36: 774-776.

19 Mattsson S, Gladh G. Tap-water enema for children with myelomeningocele and neurogenic bowel dysfunction. Acta Paediatr 2006; 95: 369-374.

20 Ausili E, Focarelli B, Tabacco F, Murolo D, Sigismondi M, Gasbarrini A et al. Transanal irrigation in myelomeningocele children: an alternative safe and valid approach for neurogenic constipation. Spinal cord 2010; 48: 560-565.

21 Pereira PL, Salvador OP, Arcas JA, Urrutia MJM, Romera RL, Monereo EJ. Transanal irrigation for the treatment of neuropathic bowel dysfunction. J Ped Urol 2010; 6: 134-138.

22 Choi EK, Shin SH, Yj Im, Kim MJ, Han SW. The effects of transanal irrigation as a stepwise bowel management program on the quality of life of children with spina bifida and their caregivers. Spinal Cord 2013; 51: 384-388.

23 Marshall DF, Boston VE. Altered bladder and bowel function following cutaneous electrical field stimulation in children with spina bifida-interim results of a randomized double-blind placebo-controlled trial. Eur J Pediatr Surg 1997; 7 (Suppl I), 41-43.

24 Palmer LS, Richards I, Kaplan WE. Transrectal electrostimulation therapy for neuropathic bowel dysfunction in children with myelomeningocele. J Urol 1997; 157: 1449-1452.

25 Han SW, Kim MJ, Kim JH, Hong CH, Kim JW, Noh JY. Intravesical electrical stimulation improves neurogenic bowel dysfunction in children with spina bifida. J Urol 2004; 171: 2648-2650.

26 Kajbafzadeh AM, Sharifi-Rad L, Nejat F, Kajbafzadeh M, Talaei HR. Transcutaneous interferential electrical stimulation for management of neurogenic bowel dysfunction in children with myelomeningocele. Int J Colorectal Dis 2012; 27: 453-458.

27 Hensle TW, Reiley EA, Chang DT. The Malone antegrade continence enema procedure in the management of patients with spina bifida. J Am Coll Surg 1998; 186: 669-674.

28 Shankar KR, Losty PD, Kenny SE, Booth JM, Turnock RR, Lamont GL et al. Functional results following the antegrade continence enema procedure. Br J Surg 1998; 85: 980-982.

29 Webb HW, Barraza MA, Stevens PS, Crump JM, Erhard M. Bowel dysfunction in spina bifida - an American experience with the ACE procedure. Eur J Pediatr Surg 1998; 8 (Suppl I), 37-38.

30 Curry JI, Osborne A, Malone PS. The MACE procedure: experience in the United Kingdom. J Pediatr Surg 1999; 34: 338-340.
31 Aksnes G, Diseth TH, Helseth A, Edwin B, Stange M, Aafos G et al. Appendicostomy for antegrade enema: effects on somatic and psychosocial functioning in children with myelomeningocele. Pediatrics 2002; 109: 484-489.

32 Dey R, Ferguson C, Kenny SE, Shankar KR, Coldicutt P, Baillie CT et al. After the honeymoon-medium-term outcome of antegrade continence enema procedure. J Pediatr Surg 2003; 38: 65-68.

33 Casale AJ, Metcalfe PD, Kaefer MA, Dussinger AM, Meldrum KK, Cain MP et al. Total continence reconstruction: a comparison to staged reconstruction of neuropathic bowel and bladder. J Urol 2006; 176: 1712-1715.

34 Lemelle JL, Guillemin F, Aubert D, Guys JM, Lottmann $H$, Lortat-Jacob S et al. A multicentre study of the management of disorders of defecation in patients with spina bifida. Neurogastroenterol Motil 2006 Feb; 18: 123-128.

35 Bani-Hani AH, Cain MP, King S, Rink RC. Tap water irrigation and additives to optimize success with the Malone antegrade continence enema: the Indiana university algorithm. J Urol 2008 Oct; 180: 1757-1760.

36 Bani-Hani AH, Cain MP, Kaefer M, Meldrum KK, King S, Johnson CS et al. The Malone antegrade continence enema: a single institutional review. J Urol 2008; 180: 1106-1110.

37 Wong AL, Kravarusic D, Wong SL. Impact of cecostomy and antegrade colonic enemas on management of fecal incontinence and constipation. Ten years of experience in pediatric population. J Pediatr Surg 2008; 43: 1445-1451.

38 Yardley IE, Pauniaho SL, Baillie CT, Turnock RR, Coldicutt P, Lamont GL et al. After the honeymoon comes divorce: long-term use of the antegrade continence enema procedure. J Pediatr Surg 2009; 44: 1274-1277.

39 Matsuno D, Yamazaki Y, Shiroyanagi Y, Ueda N, Suzuki M, Nishi M et al. The role of the retrograde colonic enema in children with spina bifida: is it inferior to the antegrade continence enema? Pediatr Surg Int 2010; 26: 529-533.

40 Bar-Yosef Y, Castellan M, Joshi D, Labbie A, Gosalbez R. Total continence reconstruction using the artificial urinary sphincter and the Malone continence enema. J Urol 2011; 185: 1444-1448.

$41 \mathrm{Ok} \mathrm{JH}$, Kurzrock EA. Objective measurement of quality of life changes after ACE Malone using the FICQoL survey. J Ped Urol 2011; 7: 389-393.

42 Siddiqui AA, Fishman SJ, Bauer SB, Nurko S. Long-term follow-up of patients after antegrade continence enema procedure. JPGN 2011; 52: 574-580.

43 Vande Velde S, Van Biervliet S, Van Laecke E, De Bruyne R, Verhelst H, Hoebeke P et al. Colon enemas in treatment of fecal incontinence in patients with spina bifida. J Urol 2013; 189: 300-304.

44 Atkins D. Grading quality of evidence and strength of recommendations. BMJ 2004; 328: 1490-1494.

45 Krogh K, Christensen P, Sabroe S, Laurberg S. Neurogenic bowel dysfunction score. Spinal Cord 2006; 44: 625-631. 